\title{
Analysis of Nonlinear Vibration of Euler-Bernoulli Beams Subjected to Compressive Axial Forcevia the Equivalent Linearization Method with a Weighted Averaging
}

\author{
Van - Hieu Dang*, Quang- Duy Le \\ Department of Mechanics, Thainguyen University of Technology, Thainguyen, Vietnam
}

*Corresponding Author: Van - Hieu Dang, Department of Mechanics, Thainguyen University of Technology, Thainguyen, Vietnam

\begin{abstract}
In this paper, the equivalent linearization method with a weighted averaging is applied to analyze nonlinear vibration of Euler-Bernoulli beam resting on the elastic foundationand subjected to the compressive axial force. Frequency-Amplitude relationships of beams with simply supported and clampedclamped end conditions are given in closed-forms. The present results are comparedwithonesachieved byusing He's Variational Approach method and the 4th-order Runge-Kutta method, comparison shows accuracy of the obtained solution. Effects of the compressive axial force and the coefficient ofelastic foundation on the nonlinear vibration behavior of beam are investigated in this work.
\end{abstract}

Keywords: equivalent linearization method, weighted averaging, nonlinear vibration, Euler-Bernoulli beam

\section{INTRODUCTION}

Vibration analysis of beams is an important issue in structural engineering applications such as long span bridges, aerospace vehicles, automobiles and many other industrial fields. The dynamics of continuous systems, such as beams, plates, and shells, are governed by nonlinear partial-differential equations in space and time. In general, it is very hard to find the exact or closed-form solutions for class of thisproblem. There are two basic points to studytype of this problem includingthe frequency domain method and the time domain method. In the time domain method, Rayleigh-Ritz and Galerkin methodsare two well-known techniques to transform partial differential equations into ordinary differential equations.

The response of nonlinear oscillation problems is fully studied by the development of approximate methods.Some approximate methods have been developed recently such asParameterized Perturbation Method (PPM) [1], Homotopy Perturbation Method (HPM) [2], Min-Max Approach (MMA)[3], Variational Iteration Method (VIM) [4], Energy Balance Method (EBM) [5] and Variational Approach (VA) [6]which were introduced by He; and the Equivalent Linearization Method (ELM) was proposed by Caughey [7].

Based on approximate analytical methods, nonlinear vibration of beams are very interested in many scientists. Using He's Variational Iteration method, free vibration problems of an Euler-Bernoulli beam under various supporting conditionswere investigated by Liu and Gurram [8]. Pakar and Payatanalyzed nonlinear vibrations of buckled Euler-Bernoulli beamsby employing He's Min-Max Approach [9].Sedighi and Reza used He's Max-Min Approach and Amplitude-Frequency Formulation to investigate effect of quintic nonlinearity on transversely vibrating of buckled EulerBernoulli beams [10]. The nonlinear dynamics of a simply supported beam resting on a nonlinear spring foundation with cubic stiffness was studied by Pellicano and Mastroddi [11]. Nonlinear vibration and postbuckling of functionally graded materials beams resting on a nonlinear elastic foundation and subjected to an axial force were studied by Yaghoobi and Torabi using the Variational Iteration method [12]. Dynamic response of an elastic beam resting on a nonlinear foundation was investigated by Younesian et al. using the Variational Iteration Method [13]. The Variational Iteration method was used by Ozturk to analyze free vibration of beam resting on elastic foundation [14]. The Homotopy Analysis method was used by Pirbodaghi et al. to investigate nonlinear vibration behaviour of Euler-Bernoulli beams subjected to axial loads [15]. Based onHe's Variational Approach and 
Laplace Iteration method, Bagheri et al. analyzed nonlinear responses of a clamped-clamped buckled beam [16]. Effect of shear deformation on free vibration of elastic beams with general boundary conditions was investigated by $\mathrm{Li}$ and Hua [17]. And recently,by using the Equivalent Linearization method, Hieu and Hai analyzed effects of the compressive axial force and the length of beams on nonlinear vibrating behaviour of quintic nonlinear Euler-Bernoulli beams subjected to axial forces [18].

Nonlinear response of a clamped-clamped buckled beam resting on linear elastic foundation was studied by Bagheri et al. [16],however, in the work, the influence of compressive axial force and coefficient of elastic foundation on vibrating response of beams has not been adequately studied.Thus, in the present paper, we focus on analyze nonlinear vibration response of Euler-Bernoulli beams resting on elastic and subjected to axial force. Frequency-Amplitude relationships of beams with simply supported (S-S) and clamped-clamped (C-C) end conditions are given in closed-forms by employing the Equivalent Linearization method with a weighted averaging. The solution is compared with the one obtained by He's Variational Approach (VA) method and the numerical one achieved by the $4^{\text {th }}$-order Runge-Kutta method. The results show accuracy of the presentsolution.Effects of the compressive axial force and the coefficient of elastic foundation on the nonlinear frequency and the frequency ratio of beam are investigated in this work.

\section{DESCRIPTION OF PROBLEM}

Consider a straight beam on an elastic foundation with length $L$, a cross-section $A$, a mass per unit length $\rho$ that subjected to an axial force of magnitude $F$ ' as shown in Figure 1. It is assumed that the cross-sectional area of the beam is uniform and its material is homogenous. With assumptions that ignore the transverse shear strains and the rotation of the cross section is due to bending only, the equation of motion of beam based on the Euler-Bernoulli beam theory as follows [19]:

$$
E I \frac{\partial^{4} w}{\partial x^{4}}+\rho \frac{\partial^{2} w}{\partial t^{2}}+\left\{F^{\prime}-\frac{E A}{2 L}\left[\int_{0}^{L}\left(\frac{\partial w}{\partial x}\right)^{2} d x\right]\right\} \frac{\partial^{2} w}{\partial x^{2}}+K^{\prime} w=0,
$$

where $K^{\prime}$ is the Winkler parameter of elastic foundation, $I$ is moment of inertia, $E$ is modulus of elasticity and wis the transverse displacement of any point on the neutral axis of beam.

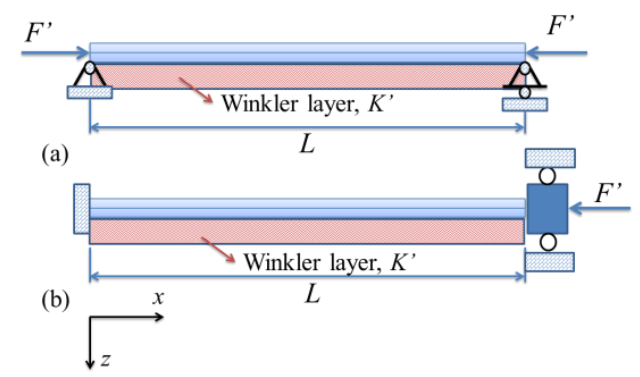

Fig1. Schematic of beams resting on the linear elastic foundation subjected to an axial load, (a)simply supported beam, (b) clamped-clamped beam

For convenience, the following nondimensional variables are introduced:

$$
\bar{x}=\frac{x}{L}, \quad \bar{w}=\frac{w}{R}, \bar{t}=t \sqrt{\frac{E I}{\rho L^{4}}}, \quad F=\frac{F^{\prime} L^{2}}{E I}, K=\frac{K^{\prime} L^{4}}{E I},
$$

where $R=(I / A)^{0.5}$ is the radius of gyration of the cross section. Using Eq. (2), Eq. (1) can be written in the dimensionless form as follows:

$$
\frac{\partial^{4} \bar{w}}{\partial \bar{x}^{4}}+\frac{\partial^{2} \bar{w}}{\partial \overline{\boldsymbol{t}}^{2}}+\left[\boldsymbol{F}-\frac{1}{2} \int_{0}^{1}\left(\frac{\partial \bar{w}}{\partial \bar{x}}\right)^{2} d \bar{x}\right] \frac{\partial^{2} \bar{w}}{\partial \bar{x}^{2}}+K \bar{w}=\mathbf{O}
$$

Assuming that displacement function $\bar{w}(\bar{x}, \bar{t})$ can be expanded as:

$$
\bar{w}(\bar{x}, \bar{t})=Q(\bar{t}) \phi(\bar{x})
$$


where $Q(\bar{t})$ is the unknown time-dependent function to be determinedand $\phi(\bar{x})$ is the basis function satisfying the kinematic boundary conditions. The basis functions are selected as follows:

+ For simply supported (S-S) beam:

$\phi(\bar{x})=\sin (\pi \bar{x})$.

(5)

+ For clamped-clamped (C-C) beam:

$\phi(\bar{x})=\frac{1}{2}[1-\cos (2 \pi \bar{x})]$.

Applying the Galerkin method, Eq. (3) is is transformed into the following ordinary differential equation:

$\ddot{Q}(\bar{t})+a_{1} Q(\bar{t})+a_{2} Q^{3}(\bar{t})=0$,

where:

$a_{1}=\frac{\int_{0}^{1} \phi^{(4)} \phi d \bar{x}}{\int_{0}^{1} \phi^{2} d \bar{x}}+F \frac{\int_{0}^{1} \phi^{\prime \prime} \phi d \bar{x}}{\int_{0}^{1} \phi^{2} d \bar{x}}+K \quad a_{2}=\frac{-\frac{1}{2} \int_{0}^{1}\left(\int_{0}^{1} \phi^{\prime 2} d \bar{x}\right) \phi " \phi d \bar{x}}{\int_{0}^{1} \phi^{2} d \bar{x}}$.

The beam is assumed to satisfy the following conditions:

$$
Q(0)=\alpha, \quad \dot{Q}(0)=0,
$$

where $\alpha$ denotes the non-dimensional maximum amplitude of oscillation.

\section{Solution Procedure}

Based on the equivalent linearization method proposed Caughey [7]; in refs. [20, 21], Anh et al. had developed this method by using the weighted averaging instead of the convenial (classical) averaging [22]. The equivalent linearization method with a weighted averaging has been used to analyze responses of strong nonlinear oscillation problems and this method gives the solutions with much better accuracy than the classical method[21, 23-25]. In this section, we will find the approximate analytical solution of Eq. (7) by using the equivalent linearization method with a weighted averaging. First, the linearizedform of Eq. (7) is introduced as follows:

$$
\ddot{Q}(\bar{t})+\omega^{2} Q(\bar{t})=0
$$

The equation error between the two oscillators given in Eq. (7) and Eq. (10) is:

$e(Q)=a_{1} Q+a_{2} Q^{3}-\omega^{2} Q$

where $\omega^{2}$ is determined by using the mean square error criterion which requires that [7]:

$$
\left\langle e^{2}(Q)\right\rangle=\left\langle\left(a_{1} Q+a_{2} Q^{3}-\omega^{2} Q\right)^{2}\right\rangle \rightarrow \underset{\omega^{2}}{\operatorname{Min}}
$$

Thus, from

$\frac{\partial}{\partial \omega^{2}}\left\langle e^{2}\right\rangle=\mathbf{O}$

we obtain:

$$
\omega^{2}=\frac{a_{1}\left\langle Q^{2}\right\rangle+a_{2}\left\langle Q^{4}\right\rangle}{\left\langle Q^{2}\right\rangle}
$$

The symbol $\langle\square$ in Eqs. (12) and (13) denotes the time-averaging operator in classical meaning [22]:

$$
\langle Q(\omega \bar{t})\rangle_{\text {classical }}=\frac{1}{T} \int_{0}^{T} Q(\omega \bar{t}) d \bar{t}=\frac{1}{2 \pi} \int_{0}^{2 \pi} Q(\tau) d \tau, \quad \tau=\omega \bar{t}
$$


where $T=2 \pi / \omega$ is the period of oscillation. The averaging value in Eq. (14) is called the classical/conventional averaging value which often leads to unacceptable errors for strongly nonlinear problems. In this paper, the weighted averaging value proposed by Anh et al. [20, 21] is used to calculate averaging values in Eq. (13). By replacing the constant coefcient $1 / T$ in Eq. (14) by a weighted coefcient function $h(\bar{t})$. By this way, the averaging value is calculated in the new way called weighted averaging value as follows:

$\langle Q(\bar{t})\rangle_{w}=\int_{0}^{+\infty} h(\bar{t}) Q(\bar{t}) d \bar{t}$.

where $h(\bar{t})$ is the weighted coefficient functionwhich must satisfies the following condition:

$\int_{0}^{+\infty} h(\bar{t}) d \bar{t}=1$.

In this paper, we use a specific form of the weighted coeficient function as [20, 21]:

$$
h(\bar{t})=s^{2} \omega \bar{t} e^{-s \omega \bar{t}},
$$

with $s$ is a positive constant, Eq. (15) will take the form of Eq. (14) when $s=0$.

The periodic solution of the linearized equation (10) is:

$$
Q(\bar{t})=\alpha \cos (\omega \bar{t})
$$

With the periodic solution given in Eq. (18) and the weighted coefficient in Eq. (17), we will calculate $\left\langle Q^{2}\right\rangle$ and $\left\langle Q^{4}\right\rangle$ by using Eq. (15), we get:

$$
\begin{aligned}
& \left\langle Q^{2}\right\rangle_{w}=\left\langle\alpha^{2} \cos ^{2}(\omega \bar{t})\right\rangle_{w}=\int_{0}^{+\infty} s^{2} \omega \bar{t} \bar{e}^{-s \omega \bar{t}} \alpha^{2} \cos ^{2}(\omega \bar{t}) d \bar{t}=\alpha^{2} \frac{s^{4}+2 s^{2}+8}{\left(s^{2}+4\right)^{2}} . \\
& \left\langle Q^{4}\right\rangle_{w}=\left\langle\alpha^{4} \cos ^{4}(\omega \bar{t})\right\rangle_{w}=\int_{0}^{+\infty} s^{2} \omega \bar{t} e^{-s \omega \bar{t}} \alpha^{4} \cos ^{4}(\omega \bar{t}) d \bar{t}=\alpha^{4} \frac{s^{8}+28 s^{6}+248 s^{4}+416 s^{2}+1536}{\left(s^{2}+4\right)^{2}\left(s^{2}+16\right)^{2}} .
\end{aligned}
$$

Subsitituting Eqs. (19) and (20) into Eq. (13), we obtain the approximate frequency:

$$
\omega=\sqrt{a_{1}+a_{2} \alpha^{2} \frac{s^{8}+28 s^{6}+248 s^{4}+416 s^{2}+1536}{\left(s^{4}+2 s^{2}+8\right)\left(s^{2}+16\right)^{2}}} .
$$

With the parameter $s$ is chosen equal to 2, the approximate frequency will be:

$$
\omega_{N L}=\sqrt{a_{1}+\frac{9216}{12800} a_{2} \alpha^{2}}=\sqrt{a_{1}+0.72 a_{2} \alpha^{2}} .
$$

Therefore, the approximate solution can be get as:

$$
Q(\bar{t})=\alpha \cos \left(\sqrt{a_{1}+0.72 a_{2} \alpha^{2}} \bar{t}\right) .
$$

\section{NUMERICAL RESULTS}

The approximate frequency $\omega_{\text {present }}$ in Eq. (22), the one obtained by Bagheri et al. using He's Variational Approach (VA) $\omega_{V A}$ as given in Eq. (24) [16] and theexact frequency $\omega_{\text {exaci }}$ is compared. It is noted that Eq. (7) is the Cubic-Duffing oscillation, the exact frequency is given in Eq. (25) [26]. Comparisonis presented in Table1for different values of the initial amplitude and $a_{l}=2, a_{2}=10$. We can see accuracy of the present solution from Table 1, when the initial amplitude of oscillation $(\alpha)$ increases,relative error of the present solution is only $0.2 \%$ while relative error of the approximate solution usingHe's Variational Approachis up to 2.2\%. 
Analysis of Nonlinear Vibration of Euler-Bernoulli Beams Subjected to Compressive Axial Forcevia the Equivalent Linearization Method with a Weighted Averaging

The approximate frequency obtanied by He's Variational Approach is given as [16]:

$\omega_{V A}=\sqrt{a_{1}+0.75 a_{2} \alpha^{2}}$.

The exact frequency of oscillation described by Eq. (7) is [26]:

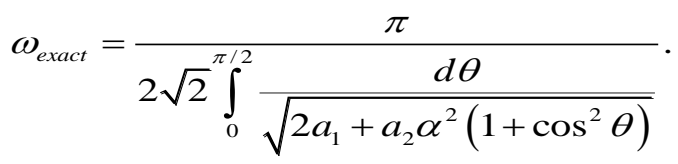

Table1. Comparison of approximate frequencies with the exact frequency

\begin{tabular}{|c|c|c|c|c|c|}
\hline$\alpha$ & $\omega_{\text {exact }}$ & $\omega_{V A}$ & R. Error (\%) & $\omega_{\text {present }}$ & R. Error (\%) \\
\hline 0.1 & 1.4404 & 1.4405 & 0.0027 & 1.4394 & 0.0696 \\
\hline 1 & 3.0411 & 3.0822 & 1.3501 & 3.0332 & 0.2628 \\
\hline 5 & 13.4734 & 13.7659 & 2.1708 & 13.4907 & 0.1286 \\
\hline 10 & 26.8302 & 27.4226 & 2.2079 & 26.8701 & 0.1484 \\
\hline 20 & 53.6619 & 54.7905 & 2.1030 & 53.6842 & 0.0415 \\
\hline 50 & 133.9639 & 136.9379 & 2.2199 & 134.1715 & 0.1549 \\
\hline 70 & 187.5442 & 191.7081 & 2.2202 & 187.8350 & 0.1551 \\
\hline 100 & 267.7916 & 273.8649 & 2.2679 & 268.3319 & 0.2017 \\
\hline
\end{tabular}

Using the basis functions given in Eqs. (5) and (6) and performing the integrations in Eq. (8), we can obtain the following expressions for the nonlinear frequency:

+ For S-S beam:

$\omega_{N L}=\sqrt{\left(\pi^{4}+K-\pi^{2} F\right)+0.72 \frac{\pi^{4}}{4} \alpha^{2}}$.

+ For C-C beam:

$\omega_{N L}=\sqrt{\left(\frac{16}{3} \pi^{4}+K-\frac{4}{3} \pi^{2} F\right)+0.72 \frac{\pi^{4}}{3} \alpha^{2}}$.

Comparison of frequency ratios $\left(\omega_{N L} / \omega_{L}\right)$ of beams achieved by two methodswith various values of the initial amplitude $(\alpha)$, the compressive axial force $(F)$ and the Winkler parameter $(K)$ are presented in Table 2.A very good agreement between the frequency ratios obtained by two methods. Noted that the linear frequency $\left(\omega_{L}\right)$ is given by:

$\omega_{L}=\sqrt{a_{1}}$.

Table2. Frequency ratios of beams achieved by two methods

\begin{tabular}{|c|c|c|c|c|c|c|}
\hline$\alpha$ & \multirow{2}{*}{$\mathrm{F}$} & $\mathrm{K}$ & \multicolumn{2}{|c|}{ S-S beam } & \multicolumn{2}{c|}{ C-C beam } \\
\cline { 3 - 7 } & & & VA [16] & Present & VA [16] & Present \\
\hline \multirow{2}{*}{0.1} & \multirow{2}{*}{10} & 10 & 1.0104 & 1.0100 & 1.0003 & 1.0003 \\
\cline { 3 - 7 } & & 50 & 1.0019 & 1.0018 & 1.0003 & 1.0003 \\
\hline \multirow{2}{*}{0.2} & \multirow{2}{*}{5} & 10 & 1.0063 & 1.0060 & 1.0010 & 1.0010 \\
\cline { 3 - 7 } & & 50 & 1.0037 & 1.0036 & 1.0010 & 1.0009 \\
\hline \multirow{2}{*}{0.5} & \multirow{2}{*}{5} & 10 & 1.0386 & 1.0371 & 1.0065 & 1.0063 \\
\cline { 3 - 7 } & & 50 & 1.0230 & 1.0221 & 1.0060 & 1.0058 \\
\hline
\end{tabular}

Comparisonsof time-responses between the present solution and the VA solution with the numerical solution using the $4^{\text {th }}$-order Runge-Kutta methodare presented in Figures 2 and 3. 


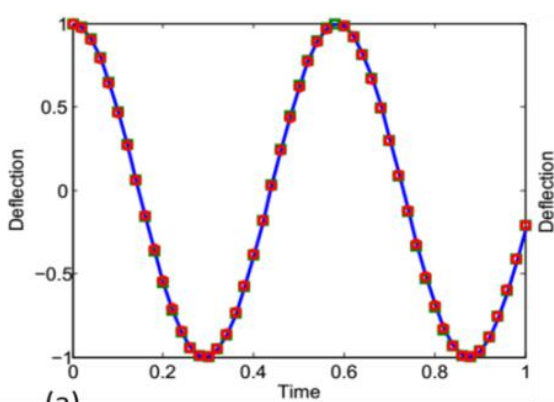

(a)

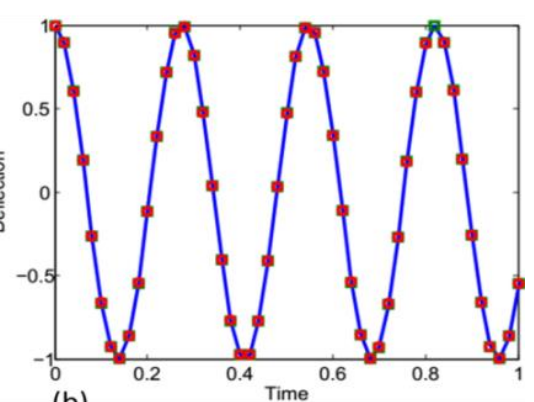

(b)

Fig2. Comparison of two approximate solutions with the numerical solutionfor $F=5, K=50$ for $(a) S$ - $S$ beam, (b) C-C beam; (line: Present solution, square : VA solution, circle: Numerical solution)

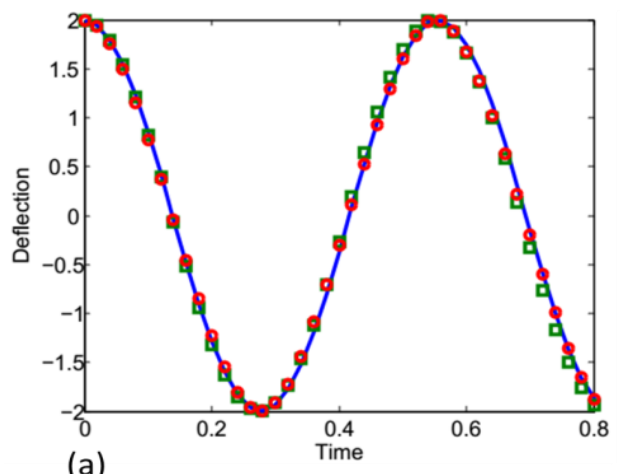

(a)

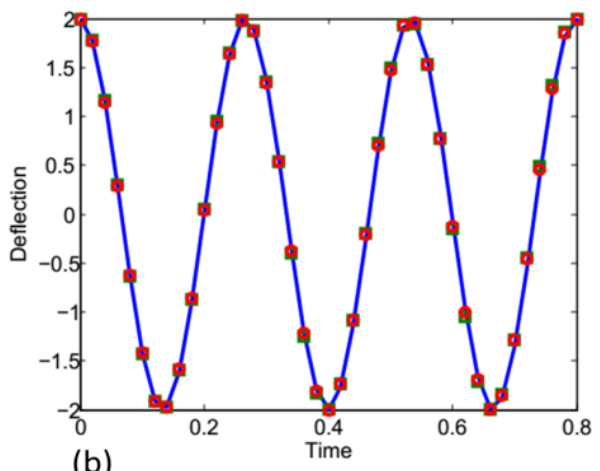

(b)

Fig3. Comparison of two approximate solutions with the numerical solution for $F=5, K=10$ for (a) $P-P$ beam, (b) C-C beam; (line: Present solution, square: VA solution, circle: Numerical solution)

Influences of the compressive axial force $(F)$ and the coefficient of elastic foundation $(K)$ on the nonlinear frequency $\omega_{N L}$ and the frequency ratio $\omega_{N L} / \omega_{L}$ are presented Figures 4-7 for both S-S and $\mathrm{C}-\mathrm{C}$ beams. Figures 4 and 5 are presented influence of the compressive axial force on vibration response of S-S and C-C beams with $K=150$, respectively. We can see from these Figures that when the initial amplitude $\alpha$ increases, both the nonlinear frequency $\omega_{N L}$ and the frequency ratio $\omega_{N L} / \omega_{L}$ of beams increase. From Figures 4 and 5, we can see that when the compressive axial force $F$ increases, the nonlinear frequency decreases; and on the other hand, the frequency ratio increases. When the compressive axial force $F$ increases, the nonlinear frequency of the beam decreases more slowly than the linear frequency, which leads to an increasing in the frequency ratio.Opposite of influence of the compressive axial force,it can be deduced from Figures 6 and 7 that the nonlinear frequency increases and the frequency ratio decreases as the Winkler parameter Kincreases. This situation is appropriate because when the coefficient of elastic foundation $K$ increases, it will make beam to harder, and therefore, the nonlinear frequency will increase. However, the increase in the nonlinear frequency is slower than the increase of the linear frequency, which will cause the frequency ratio to decrease as the coefficient of elastic foundation $K$ increases.

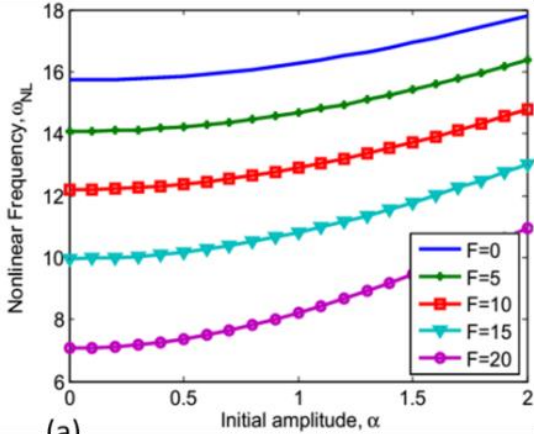

(a)

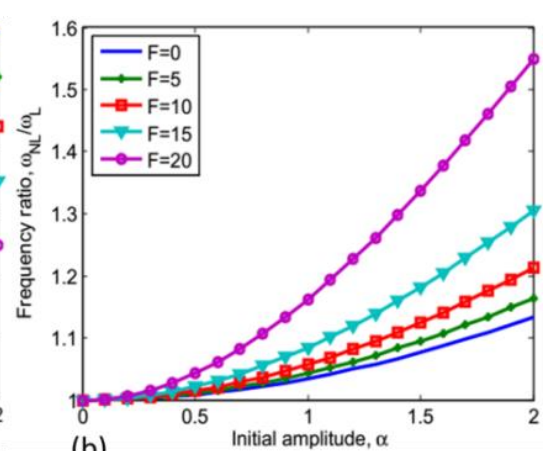

(b)

Fig4. Variation of the nonlinear frequency (a) and the frequency ratio (b) of $S$-S beam with the initial amplitude for $K=150$ 
Analysis of Nonlinear Vibration of Euler-Bernoulli Beams Subjected to Compressive Axial Forcevia the Equivalent Linearization Method with a Weighted Averaging

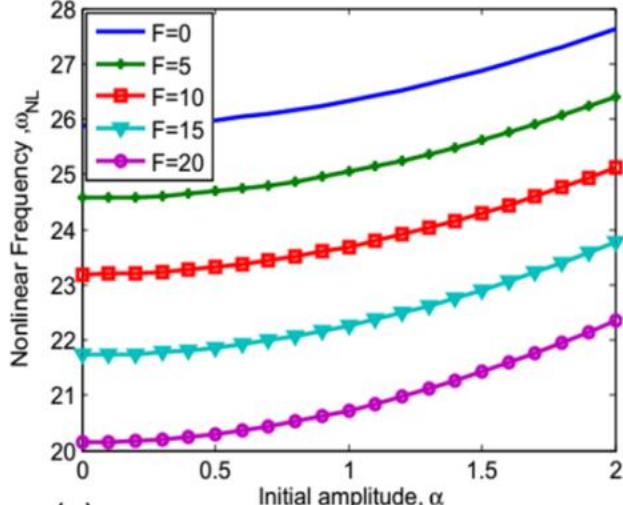

(a)

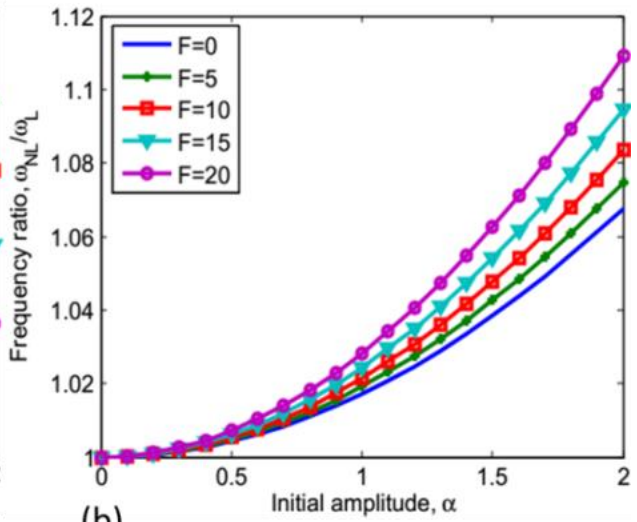

(b)

Fig5. Variation of the nonlinear frequency (a) and the frequency ratio (b) of $C$ - $C$ beam with the initial amplitude for $K=150$

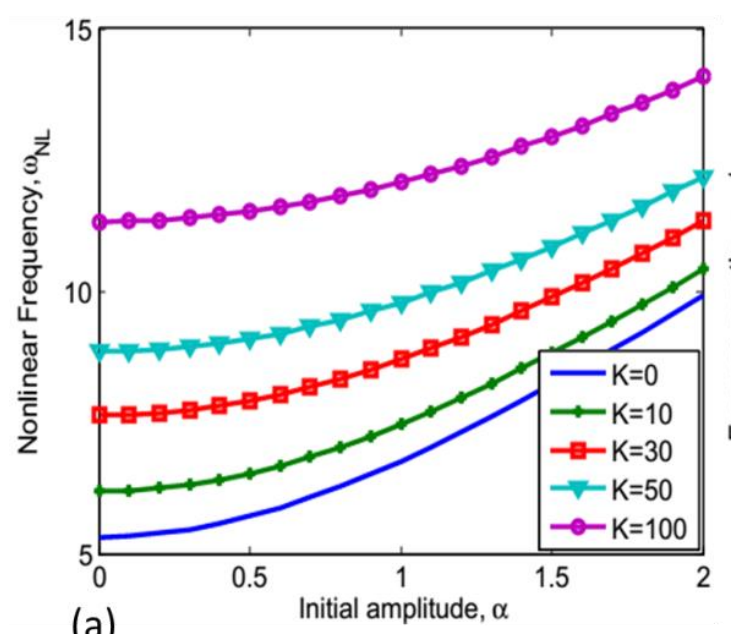

(a)

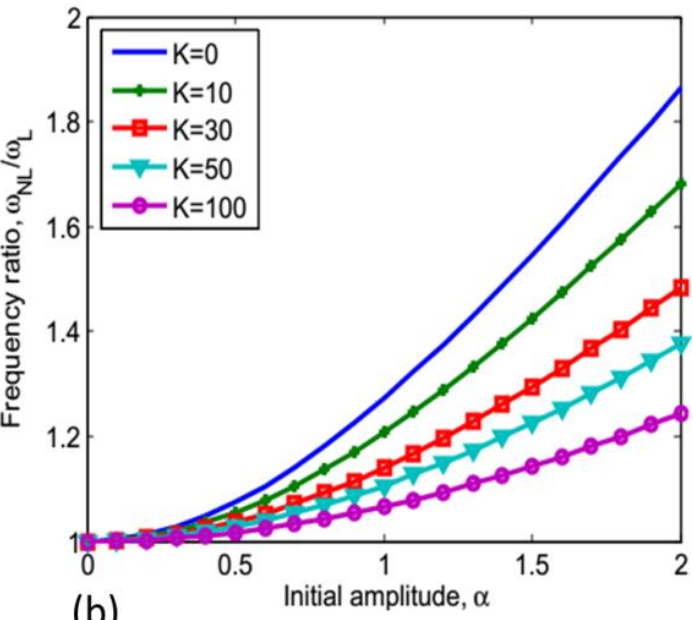

(b)

Fig6. Variation of the nonlinear frequency $(a)$ and the frequency ratio $(b)$ of $S$-S beam with the initial amplitude for $F=7$

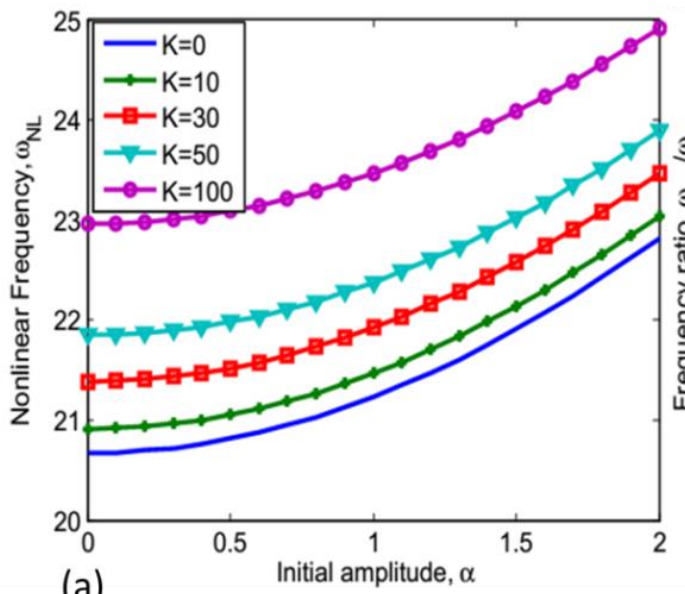

(a)

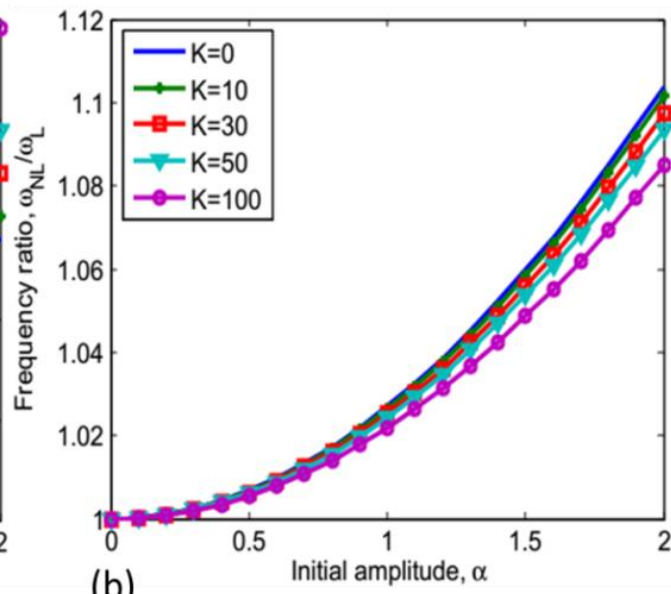

(b)

Fig7. Variation of the nonlinear frequency (a) and the frequency ratio (b) of $C$ - $C$ beam with the initial amplitude for $\mathrm{F}=7$

Futhermore, to achieve a better understanding of effects of the compressive axial force and the coefficience of elastics foundation on the vibration response of beams, we perform sensitivity analysis of thefrequencyratio via the compressive axial force, the coeficience of elastic foundation and the initial amplitue. The results of sensitivity analysis are presented in Figures 8-10. 


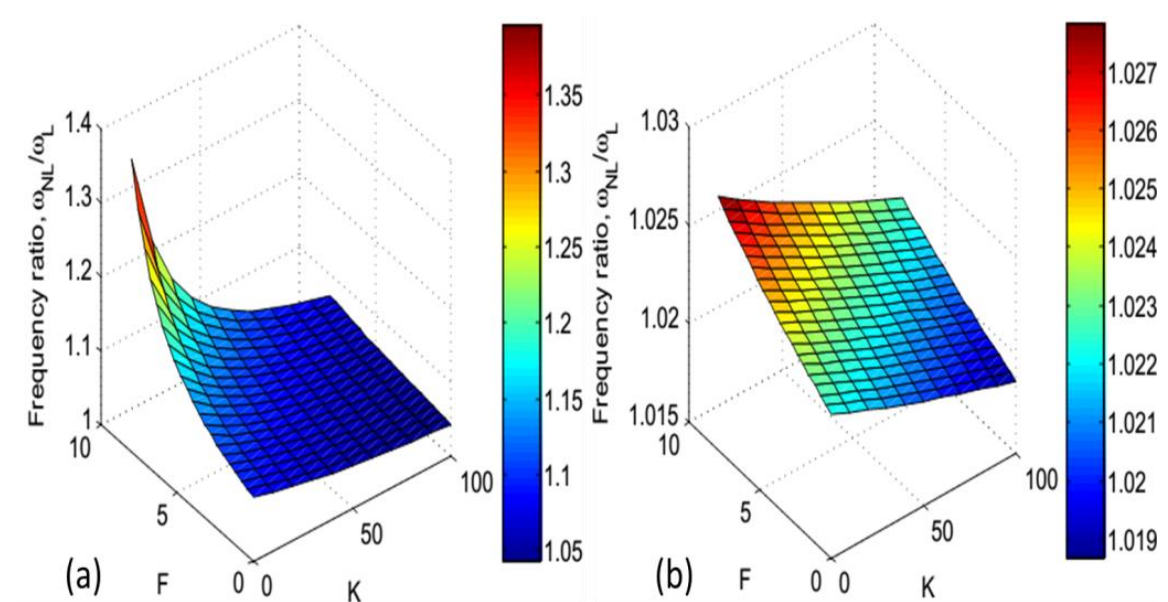

Fig8. Sensitivity analysis of the frequency ratio via the compressive axial force and the coefficient of elastic foundation with $\alpha=1$; (a) $S$-S beam, (b) C-C beam

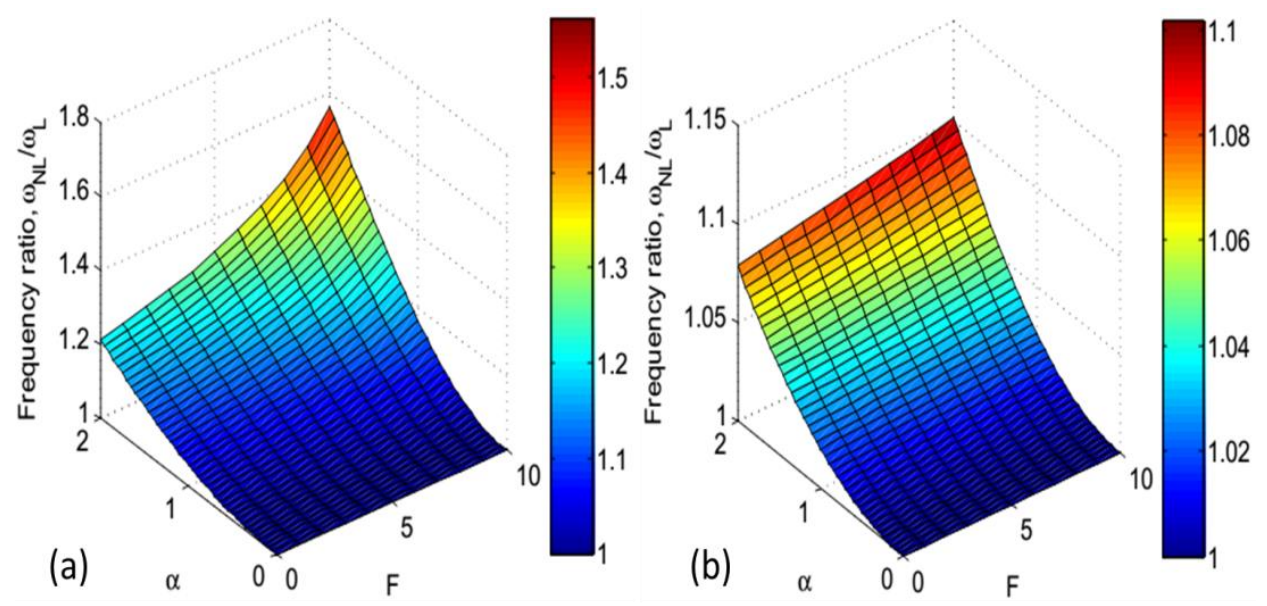

Fig9. Sensitivity analysis of the frequency ratio via the compressive axial force and the initial amplitude with $K=50$; (a) $S$-S beam, (b) $C$-C beam

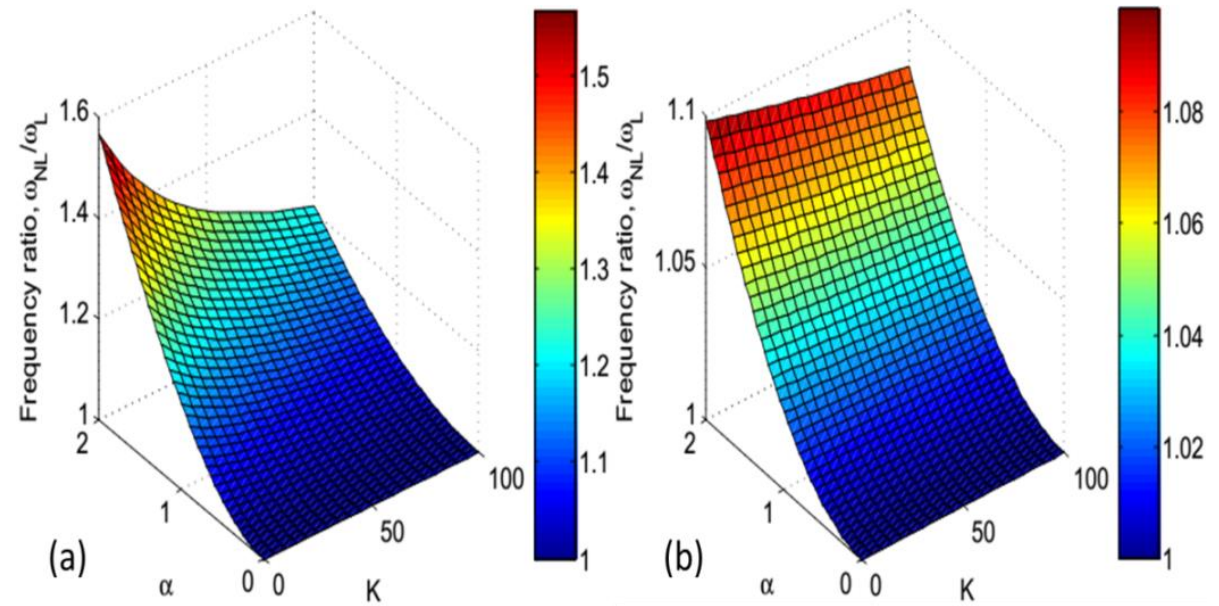

Fig10. Sensitivity analysis of the frequency ratio via the coefficient of elastic foundation and the initial amplitude with $F=5$; (a) $S$-S beam, (b) $C$-C beam

\section{CONCLUSIONS}

In this paper, nonlinear responses of Euler-Bernoulli beams resting on linear elastic foundation are investigated. The equivalent linearization method with a weighted averaging is employed to derive the amplitude - frequency relationship of beam. Comparing with the previous and numerical solutions 
shown accuracy of the present solution.Effects of the compressive axial force and the coefficient of elastic foundation on the nonlinear frequency and the frequency ratio of S-S and C-C beams are studied.

When the compressive axial force $F$ increases, the nonlinear frequency decreases; and on the other hand, the frequecy ratio increases.

The nonlinear frequency increases and the frequency ratio decreases as the Winkler parameter Kincreases.

Futhermore, sensitivity analysis of thefrequency ratio via the compressive axial force, the coeficient of elastic foundation and the initial amplitue are also investigated in this paper.

However, when the axial compressive force is very large, beams will be unstable, stable analysis of compressed axialbeams should be studied in the next time.

\section{ACKNOWLEDGEMENTS}

The research is supported by Thai Nguyen University of Technology grant for a scientific project (no. "T2018-B27”).

\section{REFERENCES}

[1] J. H. He. Some new approaches to dufng equation with strongly and high order nonlinearity (II) parametrized perturbation technique. Communications in Nonlinear Science \& Numerical Simulation,vol.4,no.1,pp.81-83,1999.

[2] J. H. He. Homotopy perturbation technique. Computer Methods Applied Mechanics and Engineering,vol.178,no.3-4,pp.257-262, 1999.

[3] J. H. He. Max-min approach to nonlinear oscillators. International Journal of Nonlinear Sciences and Numerical Simulation, vol.9 (2), pp. 207-210, 2008.

[4] J. H. He. Variational iteration method-a kind of non-linear analytical technique: Some examples. International Journal of Non-Linear Mechanics,vol.34,no.4,pp.699-708,1999.

[5] J. H. He. Preliminary report on the energy balance for nonlinear oscillations. Mechanics Research Communications, vol.29, no.2-3, pp. 107-111, 2002.

[6] J. H. He. Variational approach for nonlinear oscillators. Chaos, Solitons \& Fractals,vol.34,no.5,pp.14301439,2007 .

[7] T. K. Caughey. Equivalent linearization techniques. The Journal of the Acoustical Society of America,vol.35,pp.1706-1711, 1959.

[8] Y. Liu, C. S. Gurram. The use of He's variational iteration method for obtaining the free vibration of an Euler-Beam beam. Matematical and Computer Modelling. Vol. 50 (11-12), pp. 1545-1552, 2009.

I. Pakar and M. Payat. An analytical study of Nonlinear Vibrations of Buckled Euler - Bernoulli Beams. Acta Physica Polonica A. vol. 123, pp. 48-52, 2013.

[9] Hamid M. Sedighi, Arash Reza. The effect of quintic nonlinearity on the investigation of trensverely vibrating buckled Euler-Bernoulli Beams. Journal of Theoretical and Applied mechanics.Vol. 51(4), pp. 959-968, 2013.

[10] F. Pellicano and F. Mastroddi. Nonlinear Dynamics of a Beam on Elastic Foundation. Nonlinear Dynamics. Vol. 14, pp. 335-355, 1997.

[11] Hessameddin Yaghoobi, Mohsen Torabi. An analytical approach to large amplitude vibration and postbuckling of functionally graded beams rest on non-linear elastic foundation. Journal of Theoretical and Applied mechanics,vol. 51(1), pp. 39-52, 2013.

[12] Davood Younesian, Zia Saadatnia, Hassan Askari. Analytical solutions for oscillators of beams on nonlinear elastic doundations using the Variational Iteration Method. Journal of Theoretical and Applied mechanics, vol. 50(2), pp. 639-652, 2012.

[13] Ozturk B.. Free vibration analysis of beam on elastic foundation by the variational iteration method. International Journal of Nonlinear Sciences and Numerical Simulation, vol.10, pp. 1255-1262, 2011.

[14] T. Pirbodaghi, M.T. Ahmadian, and M. Fesanghary. On the homotopy analysis method for non-linear vibration of beams. Mechanics Research Communications, vol.36, pp.143-148, 2009.

[15] S. Bagheri, A. Nikkar, H. Ghaffarzadeh. Study of nonlinear vibration of Euler-Bernoulli beams by using analytical approximate techniques. Latin American Journal of Solids and Structures,vol.11, pp. 157 - 168, 2014. 
Analysis of Nonlinear Vibration of Euler-Bernoulli Beams Subjected to Compressive Axial Forcevia the Equivalent Linearization Method with a Weighted Averaging

[16] J. Li, H. Hua. The effects of shear deformation on the free vibration of elastic beams with general boundary conditions. Proceedings of the Institution of Mechanical Engineers, Part C: Journal of Mechanical Engineering Science, vol. 224, pp. 71-84, 2010.

[17] Dang V. Hieu, N.Q. Hai. Free Vibration Analysis of Quintic Nonlinear Beams using Equivalent Linearization Method with a Weighted Averaging. Journal of Appllied and Computional Mechanics, vol.5(1), pp. 46-57, 2019.

[18] Rao, S. S. Vibraton of Continuous Systems. John Wiley \& Sons, Inc., Hoboken, New Jersey, 2007.

[19] N. D. Anh. Dual approach to averaged values of functions: A form for weighting coefcient. Vietnam Journal of Mechanics, vol.37,no.2,pp.145-150,2015.

[20] N. D. Anh, N. Q. Hai, and D. V. Hieu. The equivalent linearization method with a weighted averaging for analyzing of nonlinear vibrating systems. Latin American Journal of Solids and Structures,vol.14,no.9,pp.1723-1740,2017.

[21] N. Minorsky. Introduction to Non-Linear Mechanics Part II: Analytical Methods of Nonlinear-Mechanics. The David W. Taylor Model Basin, United States Navy, 1945.

[22] D. Hieu and N. Hai. Analyzing of nonlinear generalized duffing oscillators using the equivalent linearization method with a weighted averaging. Asian Research Journal of Mathematics, vol.9,no.1,pp.1$14,2018$.

[23] D. V. Hieu, N. Q. Hai, and D. T. Hung. The equivalent linearization method with a weighted averaging for solving undamped nonlinear oscillators. Journal of Applied Mathematics, vol. 2018, Article ID 7487851, 15 pages, 2018.

[24] Dang Van Hieu. Postbuckling and Free Nonlinear Vibration of Microbeams Based on Nonlinear Elastic Foundation. Mathematical Problems in Engineering, Volume 2018, Article ID 1031237,17 pages.

[25] D. Younesian, H. Askari, Z. Saadatnia, M. K. Yazdi. Frequency analysis of strongly nonlinear generalized Duffing oscillators using He's frequency-amplitude formulation and He's energy balance method. Computers and Mathematics with Applications, vol. 59, pp. 3222-3228, 2010.

Citation: Hieu Dang, V\& Quang- Duy Le, (2019). Analysis of Nonlinear Vibration of Euler-Bernoulli Beams Subjected to Compressive Axial Forcevia the Equivalent Linearization Method with a Weighted Averaging. International Journal of Scientific and Innovative Mathematical Research (IJSIMR), 7(1), pp.413. http://dx.doi.org /10.20431/2347-3142.0701002

Copyright: (c) 2019 Authors, This is an open-access article distributed under the terms of the Creative Commons Attribution License, which permits unrestricted use, distribution, and reproduction in any medium, provided the original author and source are credited. 